INFORME DE PRACTICA EMPRESARIAL - DIRECCION SECCIONAL DE IMPUESTOS Y ADUANAS DE VILLAVICENCIO - DIAN.

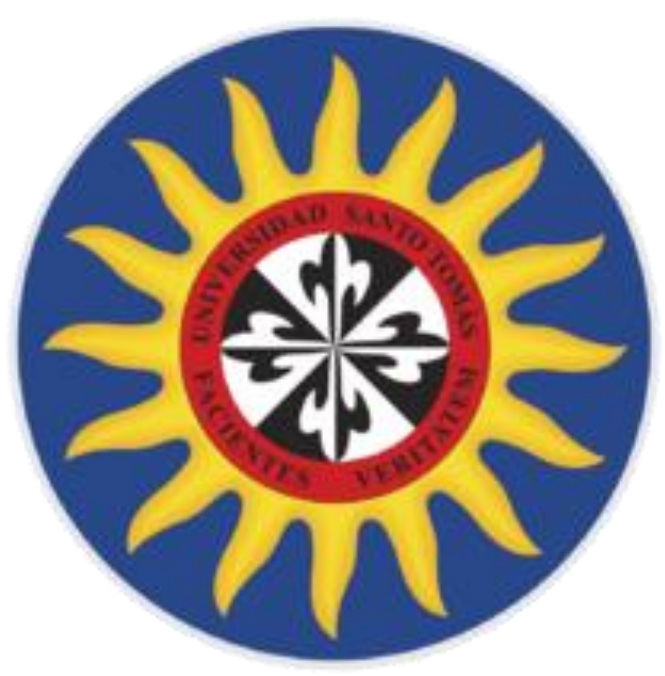

OSCAR ALIRIO BENITO MOLINA

UNIVERSIDAD SANTO TOMAS

FACULTAD DE CONTADURÍA PÚBLICA

VILLAVICENCIO

2018 


\title{
INFORME DE PRACTICA EMPRESARIAL - DIRECCION SECCIONAL DE IMPUESTOS Y ADUANAS DE VILLAVICENCIO - DIAN.
}

\section{OSCAR ALIRIO BENITO MOLINA}

Informe de práctica presentado como requisito para optar al título de Contador Público

\author{
Asesor \\ CELIA CRISTINA CAÑAS OLIVO \\ Contador Público
}

\author{
UNIVERSIDAD SANTO TOMAS \\ FACULTAD DE CONTADURÍA PÚBLICA \\ VILLAVICENCIO
}

2018 
Autoridades Académicas

P JUAN UBALDO LÓPEZ SALAMANCA, O.P.

Rector General

\section{P MAURICIO ANTONIO CORTES GALLEGO, O.P.}

Vicerrector Académico General

P JOSÉ ARTURO RESTREPO RESTREPO, O.P.

Rector Sede Villavicencio

P FERNANDO CAJICÁ GAMBOA, O.P.

Vicerrector Académico Sede Villavicencio

Adm. JULIETH ANDREA SIERRA TOBÓN

Secretaria de División Sede Villavicencio

Dr. JOSÉ JIMMY SARMIENTO MORALES

Decano de la Facultad de Negocios Internacionales 


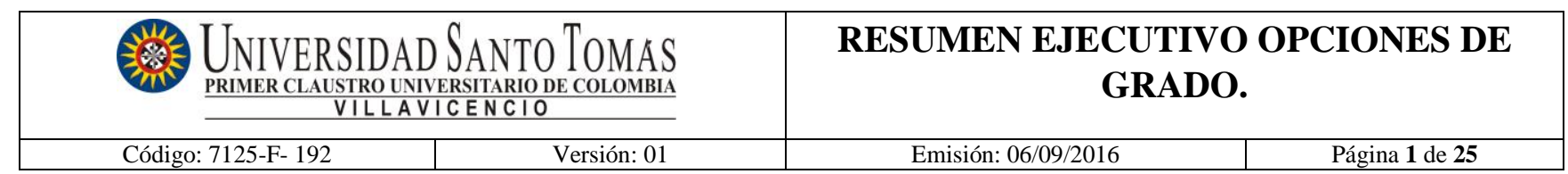

TABLA DE CONTENIDO

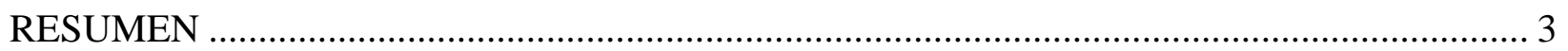

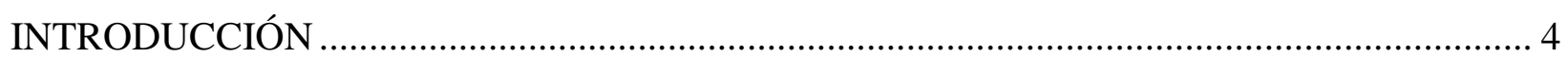

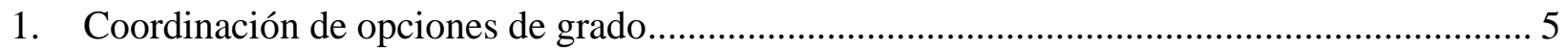

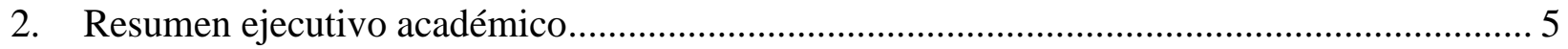

2.1. Proyecto o Actividad desarrollada objeto de informe manejada por el equipo ................ 5

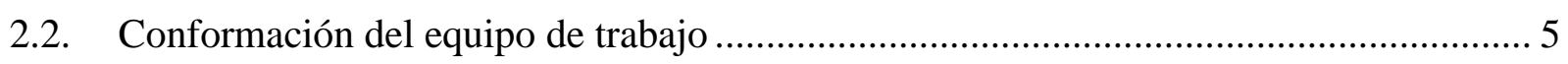

2.3. Universidad vinculada en el desarrollo del proyecto o actividad....................................... 6

2.4. Facultad vinculada en el desarrollo del proyecto o actividad .......................................... 6

2.5. Empresa vinculada en el desarrollo del proyecto o actividad área funcional específica de

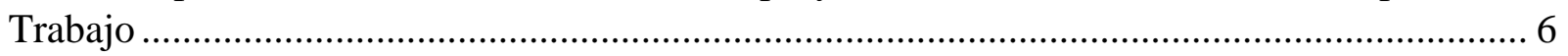

2.6. Ciudad y fecha de realización del Informe................................................................... 6

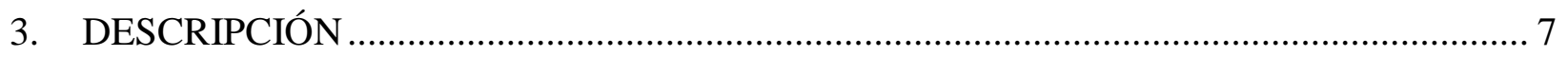

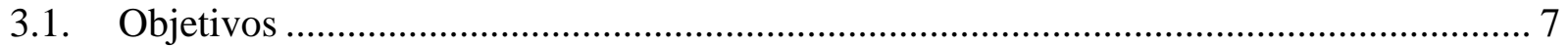

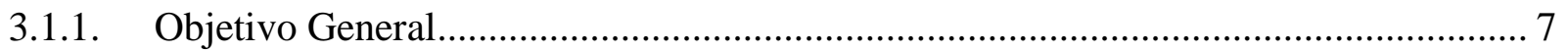

3.1.2. Objetivos Específicos ………………………............................................... 7

3.1.3. Objetivos Práctica.................................................................................................. 7

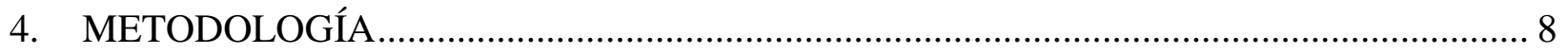

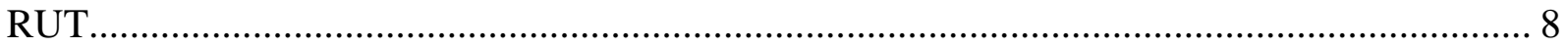

Firma Electrónica (F.E.) ………………………………............................................. 9

Autorización Numeración de Facturación ................................................................................. 9

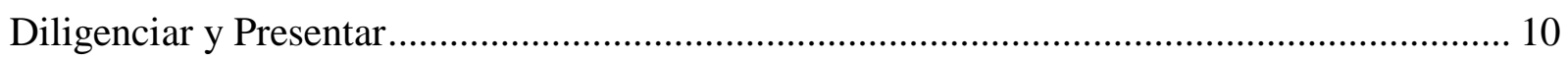

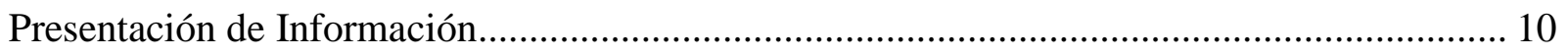

Consultar Información Suministrada Por Terceros................................................................ 11

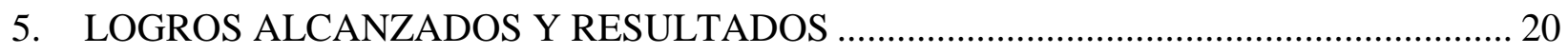

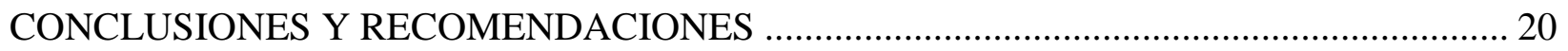

REFERENTES BIBLIOGRAFICOS, VIDEOS, WEB Y CON EXPERTOS ………………...... 21

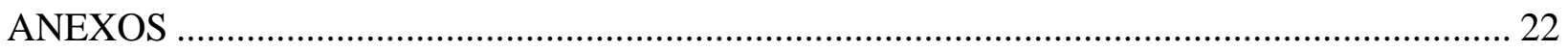




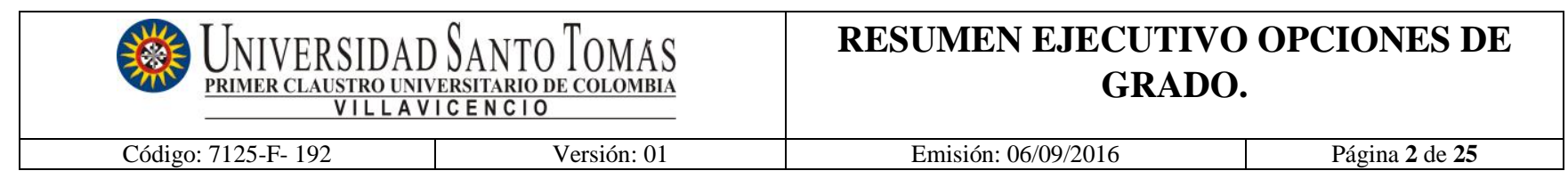

LISTA DE ILUSTRACIONES

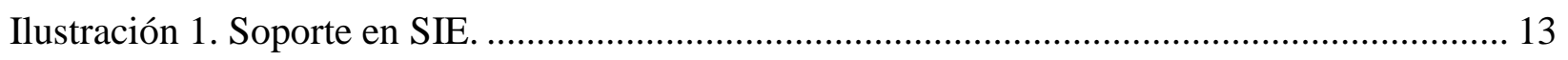

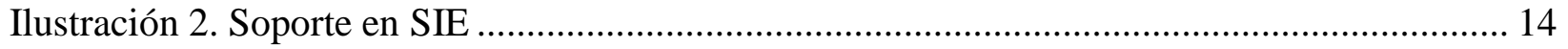

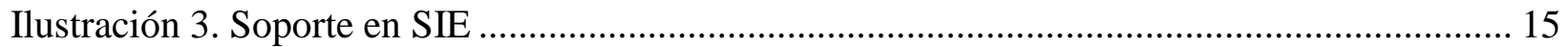

Ilustración 4. Manejo Usuario Autorizado............................................................................ 16

Ilustración 5. Error habilitando Firma Electrónica .................................................................. 17

Ilustración 6. Error habilitando cuenta de usuario ....................................................................... 18

Ilustración 7. Capacitación Responsabilidades RUT............................................................. 19 


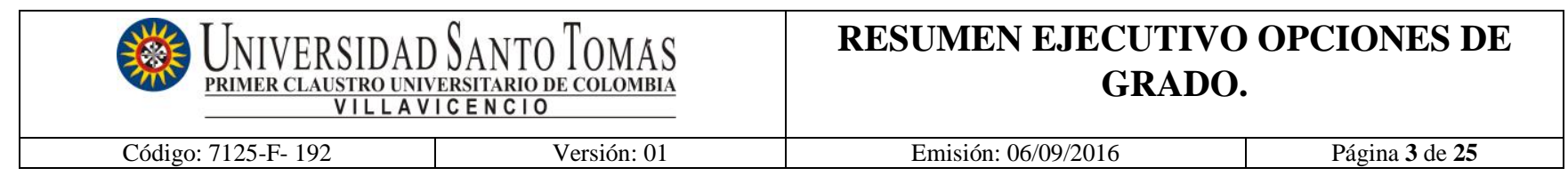

\section{RESUMEN}

En el trabajo se presentará los servicios informáticos que ofrece la DIAN, los cuales se tuvo que aprender a manejar para poder brindarles una correcta asesoría, respecto al manejo de la plataforma, a los contribuyentes que llegasen al punto de contacto, además temas de algunas capacitaciones en las cuales nos hicimos participes para dar a los contribuyentes soporte respecto al proceso de los distintos trámites.

\section{Palabras clave: DIAN, Servicios Informáticos, contribuyente}




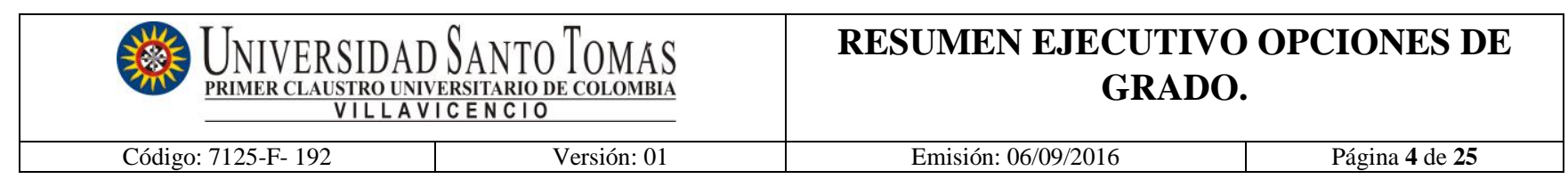

\section{INTRODUCCIÓN}

En trabajo en la división de Gestión De Asistencia al cliente, se evidencia un entendimiento, no solo los temas referentes a todos los Servicios Informáticos Electrónicos, SIE, de la DIAN, sino también un manejo y trato en la asistencia hacía los contribuyentes.

En la opción de grado, Práctica empresarial, realizada en la Unidad Administrativa Especial Dirección de Impuestos y Aduanas Nacionales, U.A.E. DIAN, se busca aplicar los conocimientos adquiridos en materia tributaria durante el proceso académico y llevarlos a la práctica real, además de tener una visión del mundo fuera del aula de clase. 


\begin{tabular}{|c|c|}
\hline$\frac{\text { 㳑: }}{\frac{\text { UNIMER CLAUSTRO UNIVERSITARIO DE COLOMBIA }}{\text { VILLAVIC E N C IO }}}$ & $\begin{array}{l}\text { RESUMEN EJECUTIVO OPCIONES DE } \\
\text { GRADO. }\end{array}$ \\
\hline Código: 7125-F- 192 & Emisión: 06/09/2016 \\
\hline
\end{tabular}

1. Coordinación de opciones de grado

Tipo De Actividad

Marque con una $(\mathrm{X})$ la opción de grado.

Curso de Grado

Trabajo de Grado

Preparatorios

Promedio Ponderado

Trabajo Social X

\section{Resumen ejecutivo académico}

\subsection{Proyecto o Actividad desarrollada objeto de informe manejada por el equipo}

Trabajo social desarrollado en la Dirección de Impuestos y Aduanas Nacionales (DIAN).

\subsection{Conformación del equipo de trabajo}

Funcionarios: Ricardo Gutiérrez Pachón (Jefe de división), Silvia Elena Correa Castillo (Jefe directa), Gildardo Vanegas (Orientador), Eliseo Ramírez (Encargado de solicitudes especiales), (Julián Andrés Bustos (Rut, F.E., etc.), Yolanda Garzón (Rut, F.E., etc.), Ramiro Conde (Rut, F.E., etc.), Ismael Perdomo (Rut, F.E., etc.), Carmen Tulia Parrado (Libros y Auditorias), Martha López 


\begin{tabular}{|c|c|c|c|}
\hline 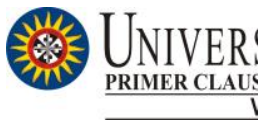 & $\begin{array}{l}0 \text { TOMAS } \\
0 \\
0\end{array}$ & \multicolumn{2}{|c|}{$\begin{array}{l}\text { RESUMEN EJECUTIVO OPCIONES DE } \\
\text { GRADO. }\end{array}$} \\
\hline Código: 7125-F- 192 & Versión: 01 & Emisión: 06/09/2016 & Página 6 de 25 \\
\hline
\end{tabular}

(Rut, F.E., etc.), Jazmín Vega (Rut, F.E., etc.), Yulied Oviedo (Rut, F.E., etc.), Marcela Gualteros

Ossa (Atención en Kiosco).

Pasantes: Mónica Tatiana Guavita (Contaduría Pública), Angie Yubelly Bejarano (Negocios Internacionales), Natalia Medina Figueroa (Negocios Internaciones)

\subsection{Universidad vinculada en el desarrollo del proyecto o actividad}

Universidad Santo Tomás, Sede Villavicencio.

\subsection{Facultad vinculada en el desarrollo del proyecto o actividad}

Programa de Contaduría Pública.

\subsection{Empresa vinculada en el desarrollo del proyecto o actividad área funcional específica de Trabajo}

Dirección de Impuestos y Aduanas Nacionales de Colombia (DIAN).

\subsection{Ciudad y fecha de realización del Informe}

Villavicencio, XX de XX de 2018. 


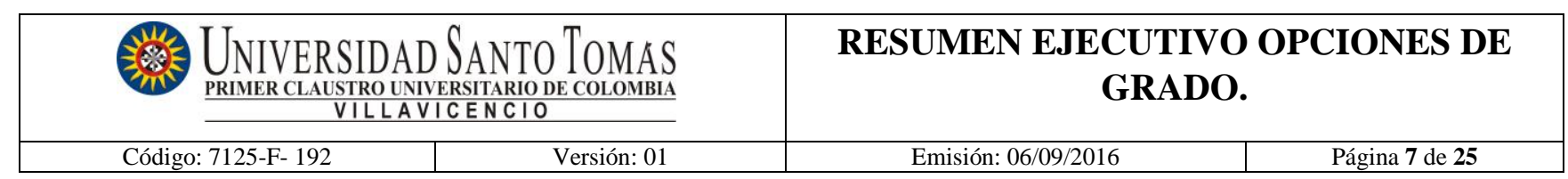

\section{DESCRIPCIÓN}

\subsection{Objetivos}

\subsubsection{Objetivo General}

- Comprender el funcionamiento de los servicios informáticos que maneja la DIAN.

\subsubsection{Objetivos Específicos}

- Conocer el correcto manejo de la plataforma de la DIAN, MUISCA.

- Obtener el conocimiento necesario para responder las dudas de los contribuyentes.

- Mantener una correcta actualización en materia tributaria.

\subsubsection{Objetivos Práctica}

- Cumplir Satisfactoriamente con el requisito de grado

- Tener una visión cercana al área laboral y no solo del aula de clase 


\begin{tabular}{|c|c|c|c|}
\hline 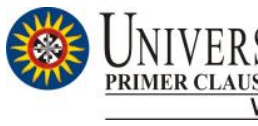 & $\begin{array}{l}0 \text { TOMAS } \\
0 \\
0\end{array}$ & \multicolumn{2}{|c|}{$\begin{array}{l}\text { RESUMEN EJECUTIVO OPCIONES DE } \\
\text { GRADO. }\end{array}$} \\
\hline Código: 7125-F- 192 & Versión: 01 & Emisión: 06/09/2016 & Página 8 de 25 \\
\hline
\end{tabular}

\section{METODOLOGÍA}

La práctica se inició en la División de Gestión de asistencia al cliente, en el área del Kiosco, lugar donde llegan todos los contribuyentes para contar con asesoría respecto a los trámites relacionados con la DIAN.

En este sitio se debió aprender a manejar los distintos servicios informáticos electrónicos (SIE) que ofrece la DIAN, para esto se contó con la ayuda de la funcionaria Marcela Gualteros, persona encargada de capacitarnos durante las dos primeras semanas después de haber iniciado las prácticas.

Para poder empezar a hablar de los SIE de la DIAN es necesario hacer la aclaración que, en primera instancia, se debe estar inscrito en el Registro Único Tributario, RUT, si no se está inscrito no se podrá acceder, y en segunda, contar con la clave de acceso, que es establecida por el mismo contribuyente, si el contribuyente no cuenta con la clave se puede recuperar por medio del correo o acercándose al punto de contacto y solicitar un "reseteo de cuenta". Teniendo la clave y el usuario se puede ingresar a los SIE; A continuación, se va a hablar acerca de los SIE aplicados durante el tiempo de duración de la pasantía;

\section{RUT}

En este SIE se adquirieron conocimientos acerca de los requisitos necesarios para poder inscribir el RUT de las personas naturales o jurídicas, en este último se hace necesario conocer la naturaleza de la misma, es decir, si es comercial, de carácter social, si es de algún consorcio o unión temporal, etc. 


\begin{tabular}{|c|c|c|c|}
\hline 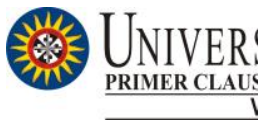 & $\begin{array}{l}0 \text { TOMAS } \\
0 \\
0\end{array}$ & \multicolumn{2}{|c|}{$\begin{array}{l}\text { RESUMEN EJECUTIVO OPCIONES DE } \\
\text { GRADO. }\end{array}$} \\
\hline Código: 7125-F- 192 & Versión: 01 & Emisión: 06/09/2016 & Página 9 de 25 \\
\hline
\end{tabular}

También se aprendió a actualizar el RUT, que es necesario poder abrir un establecimiento, prestar un servicio, adquirir un contrato e incluso el recibir el pago de la empresa donde se labore, hay que aclarar que existen ítems en los cuales se hace necesario agendar una cita para actualizar, por ejemplo, el nombre.

De igual modo se conoce el procedimiento a seguir cuando una empresa que haya terminado su funcionamiento, pueda cerrar ante la DIAN, que consta, primero, de actualizar el RUT y solicite la cancelación del establecimiento por medio de la creación de una "Solicitud especial".

\section{Firma Electrónica (F.E.)}

Por medio de esta F.E. las personas jurídicas pueden cumplir con sus obligaciones sustanciales, como el de presentar declaraciones, además de otros servicios como actualizar su RUT, presentar información por medio de prevalidadores, de los cuales se hablará más adelante, en general, cualquier trámite por medio de los SIE necesitará de la FE, se aclara que el instrumento de la F.E. pertenece a una persona natural, ya sea el representante legal, revisor fiscal, etc., y es esta persona natural es quien debe hacer el proceso personalmente.

Aquí se debe aclarar que una vez una persona natural adquiere el instrumento de F.E. por cualquier motivo, por ejemplo, haber sido representante legal de una empresa, debe estarla renovando cada dos años.

\section{Autorización Numeración de Facturación}

Es con esta autorización que los contribuyentes y/o declarantes pueden autenticar sus facturas, para este proceso es necesario que se cuente con la F.E., vale resaltar que quienes pertenecen al 


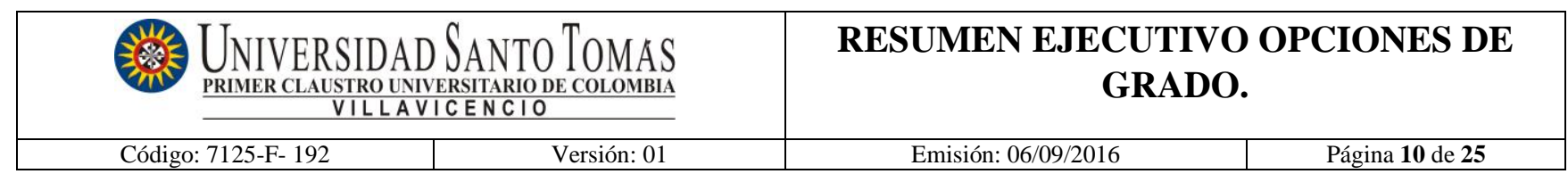

régimen simplificado no son obligados a facturar, si alguien perteneciente a este régimen quiere solicitar la Numeración se debe averiguar el motivo por el cual la está solicitando.

\section{Diligenciar y Presentar}

Desde esta opción los contribuyentes todas las personas, naturales o jurídicas, deben presentar sus las declaraciones a las que haya lugar. Para poder presentar una declaración por este medio es necesario que el contribuyente cuente con la F.E., en caso de no tenerla debe presentarla litográficamente en los bancos; el que una persona natural no cuente con firme electrónica no lo impide poder diligenciar las declaraciones por este medio, lo puede hacer, pero una vez realizada la debe presentar y pagar en el banco.

\section{Presentación de Información}

Esta es la función asignada para suministrar la información por medio de los prevalidadores, estos permiten colocar toda la información contable. Estos prevalidadores son brindados por la U.A.E. DIAN en su portal web, el contribuyente debe realizar y validar según su respectiva información contable, para poder subir la información al portal, la DIAN dispone de distintas funciones, como lo son, "Colocar Archivos en su Bandeja de Entrada", este es el primer paso, absolutamente todos los prevalidadores deben ser colocados en la bandeja de entrada, para luego poderlos diligenciar; el siguiente paso es "Diligenciar Solicitud Envío de Archivos", es aquí donde se digita los aspectos generales del prevalidador como los son, Año que se informa, cantidad de registros que contiene el mismo, el nombre y la cantidad de archivos.

Además el SIE ofrece las opciones de "Consultar envíos de solicitudes" en la cual se puede confirmar que el prevalidador haya quedado adjuntado de forma correcta y no presente errores; En 


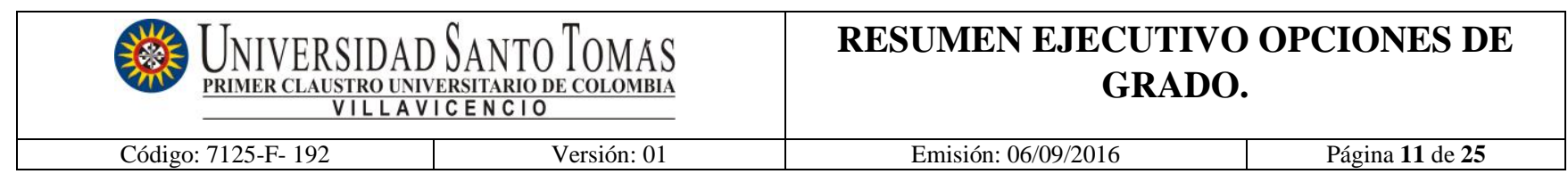

caso de que el prevalidador presente errores el SIE ofrece dos opciones, la primera es, en caso de que se presente un error de presentación, que son inconsistencias en los aspectos generales del prevalidador, que se mencionaron más arriba, se debe utilizar la opción "Reemplazar Solicitud de Envío de Archivos", la cual pretende hacer el nuevo envío con la posibilidad de corregir los errores de diligenciamiento hallados, la segunda opción es "Reemplazar Parcialmente Envío de Archivos" la cual se usará cuando se presenten errores en la realización del prevalidador, un error frecuente de este tipo es confundir el tipo de identificación, cédula de ciudadanía con NIT, con la opción mencionada se puede presentar nuevamente, corrigiendo el error encontrado.

\section{Consultar Información Suministrada Por Terceros}

Por medio de este SIE las personas naturales pueden consultar cualquier movimiento financiero con que un tercero, persona natural o jurídica, lo esté vinculando, lo que se quiere lograr con esto es que las personas naturales puedan saber si tienen la obligación formal de presentar declaración de renta sin la obligación o necesidad de acercarse al punto de contacto.

De igual forma se nos enseñó el manejo de las plataformas usadas por los funcionarios, para esto se usó el usuario de la Jefe directa Silvia Correa, desde aquí se verificaba estado en el que se encuentra un RUT y firma electrónica, también el control de turnos, enviar errores que, por funcionamiento interno de la DIAN, deben ser resueltos directamente desde Bogotá.

Los contribuyentes también llegaban con dudas acerca de cualquier índole, para poder darles una correcta orientación se recibía capacitaciones, hechas internamente por el Orientador Gildardo Vanegas, aquí se aclaraba cualquier duda que se tuviese. 


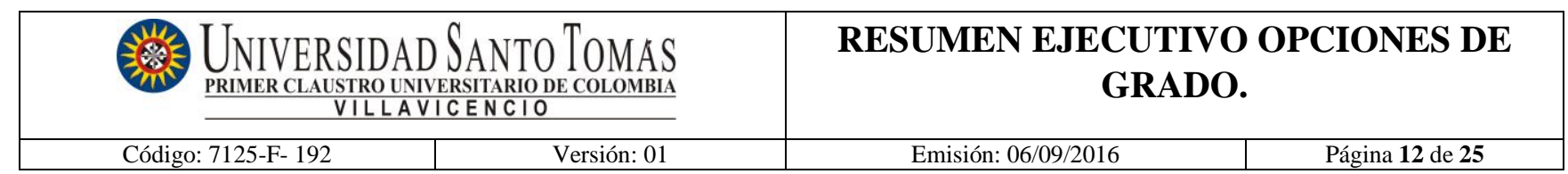

En el Kiosco se evitaba realizar los procesos por el contribuyente, sino por el contrario se les orientaba para que, en un futuro, se pudiesen evitar las molestias o inconvenientes de acercarse al punto de contacto, se les enseñaba los pasos y la forma de hacer los trámites por los cuales se acercaban, aunque, existen algunos trámites para los que se hace necesario la necesidad de acercarse al punto de contacto con cita programada, pero dependiendo la situación, en la mayoría de casos es que tenga vencimiento el mismo día del inconveniente, se debe acercar sin cita previa.

Además, al final del día se hacía una retroalimentación con los compañeros acerca de los casos especiales que se habían presentado durante el día para poderlos solucionar en caso que se vuelvan a presentar. 


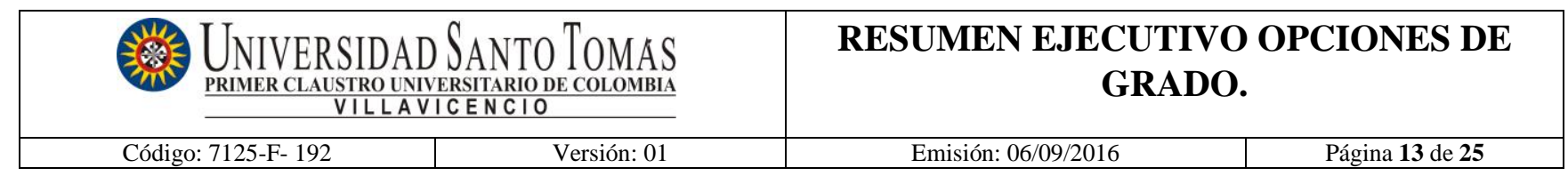

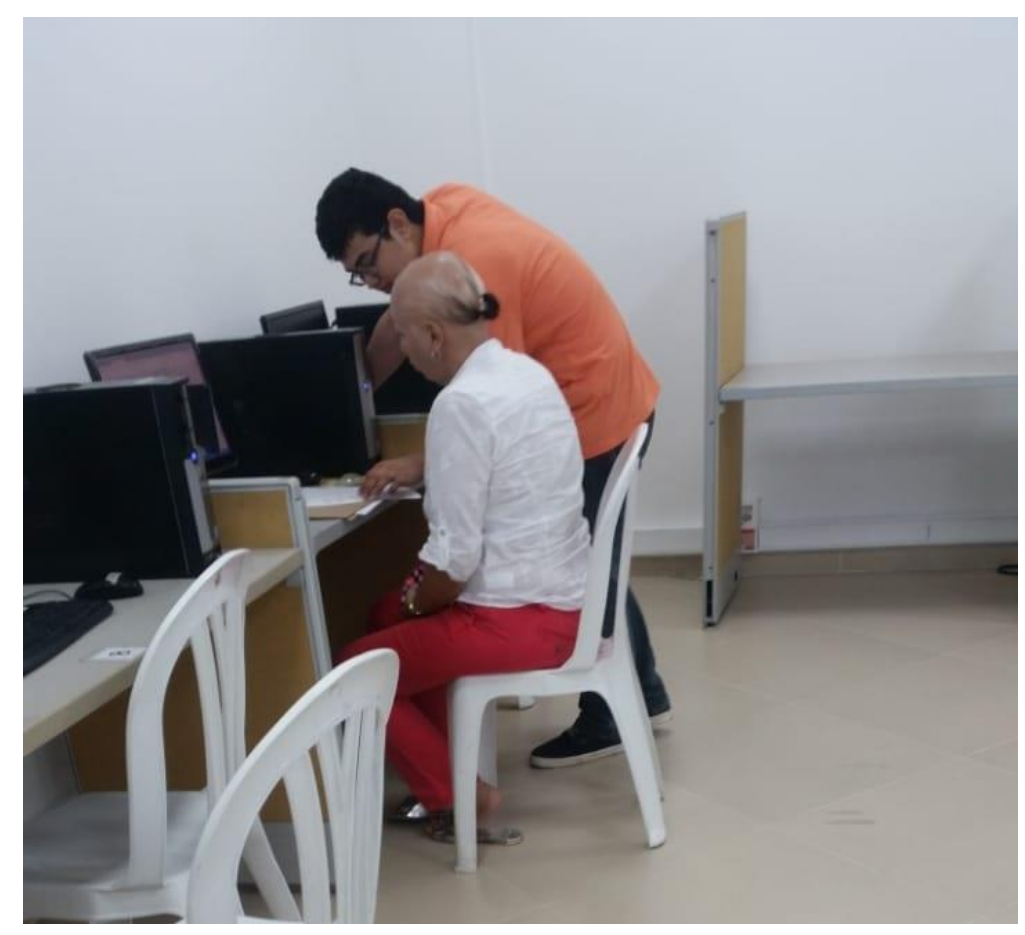

Ilustración 1. Soporte en SIE. 


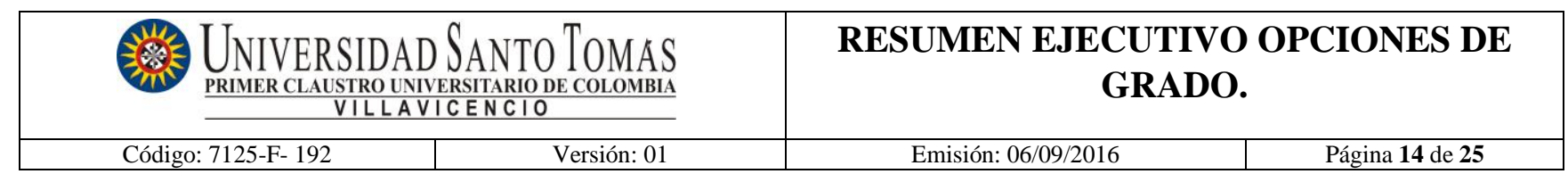

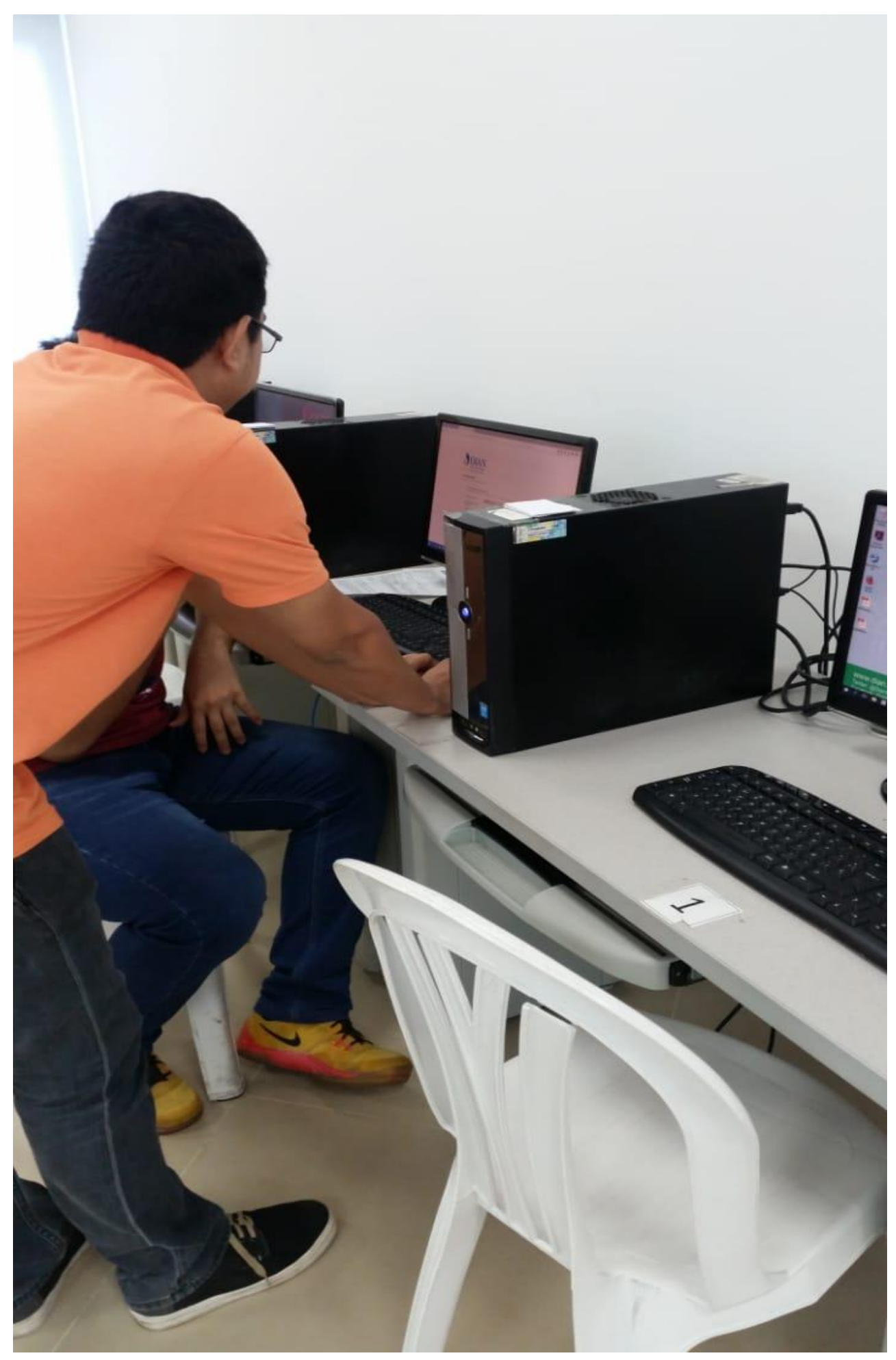

Ilustración 2. Soporte en SIE 


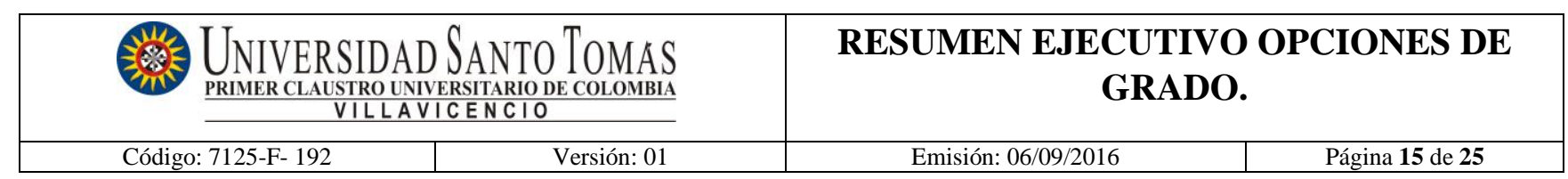

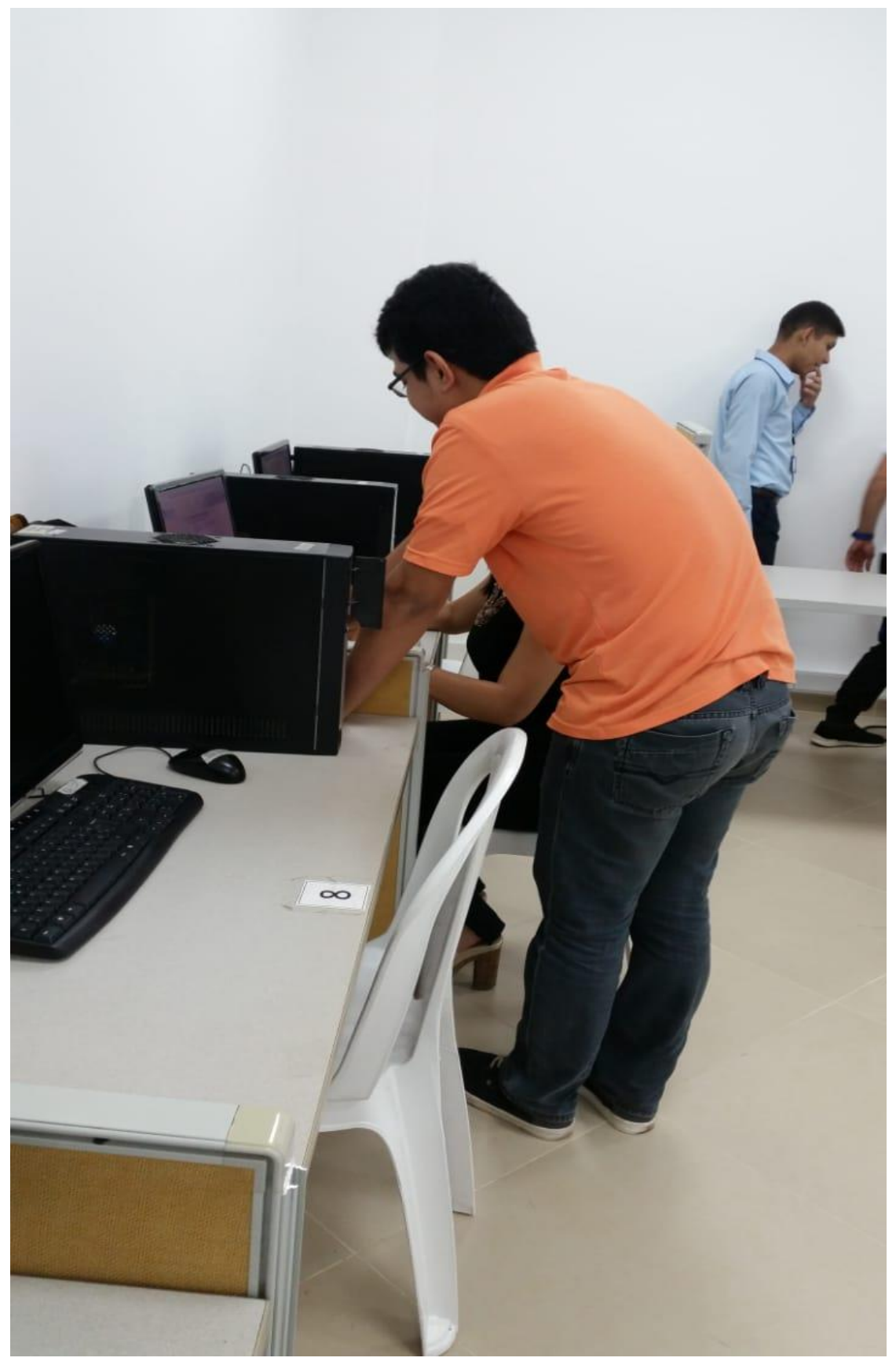

Ilustración 3. Soporte en SIE 


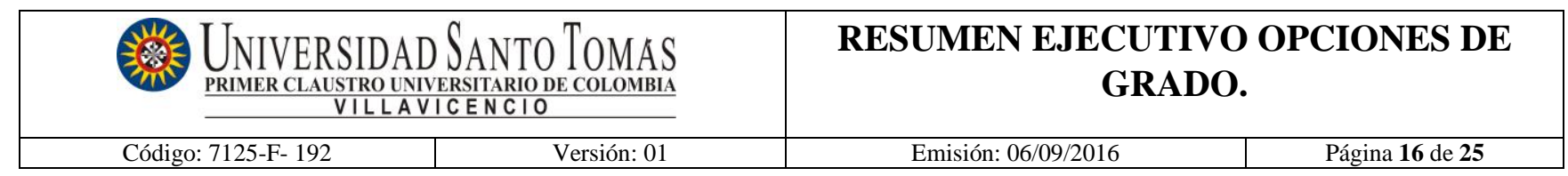

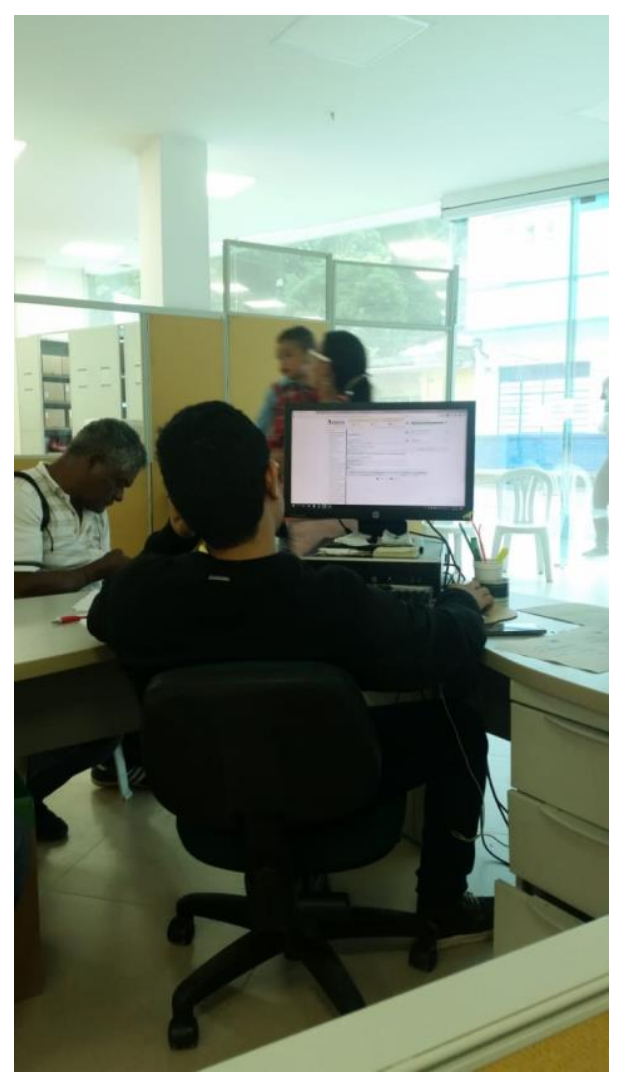

Ilustración 4. Manejo Usuario Autorizado 


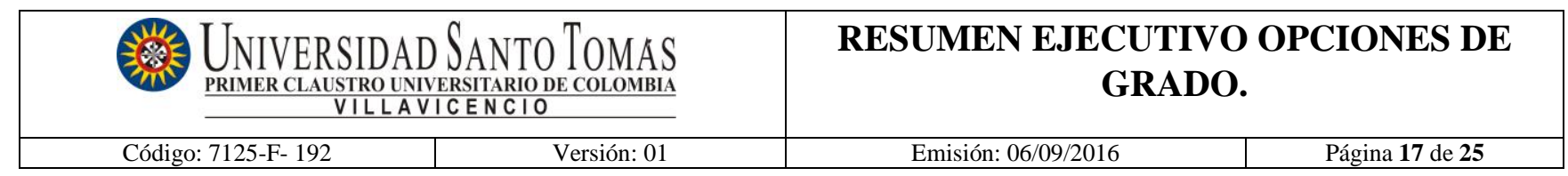

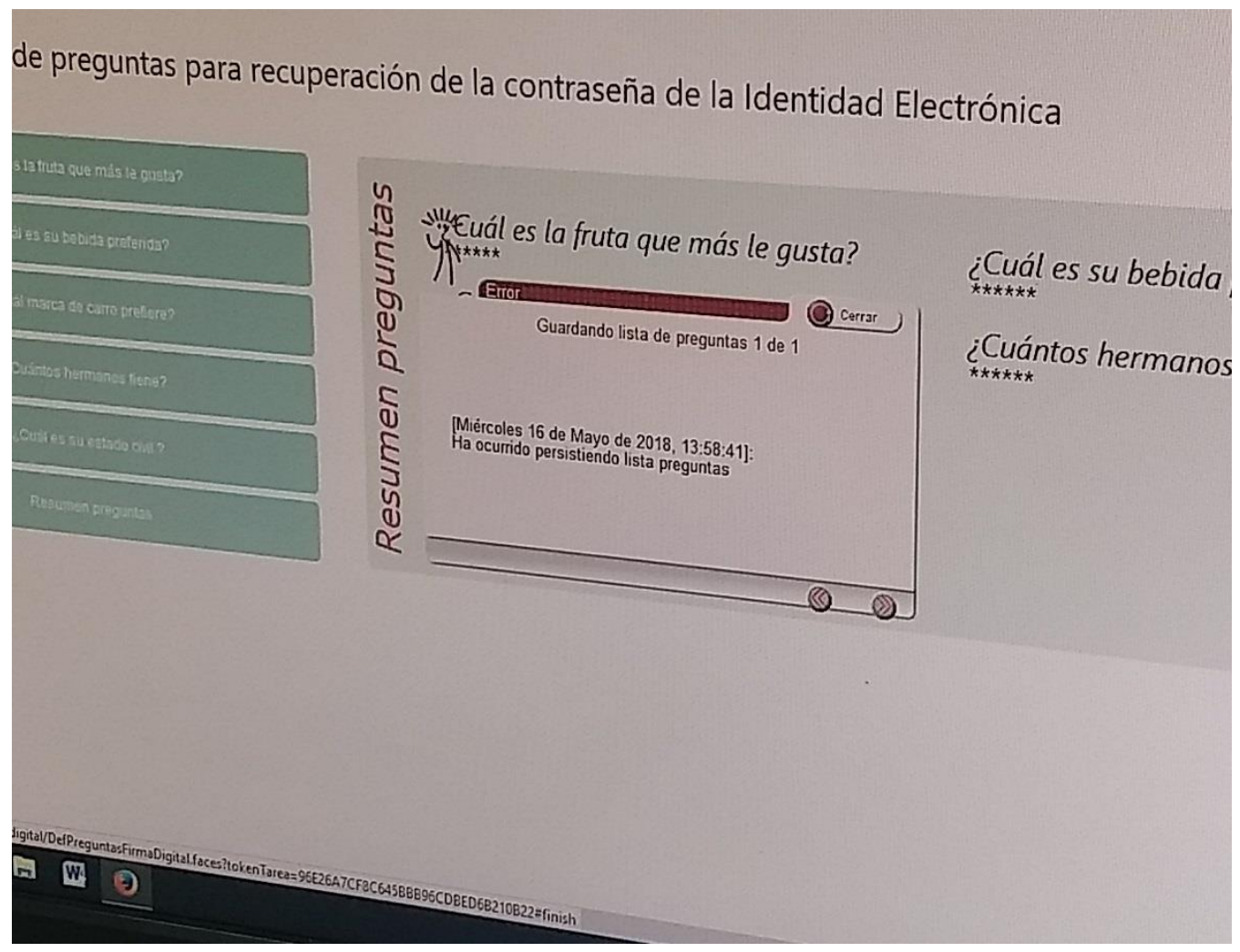

\section{Ilustración 5. Error habilitando Firma Electrónica}




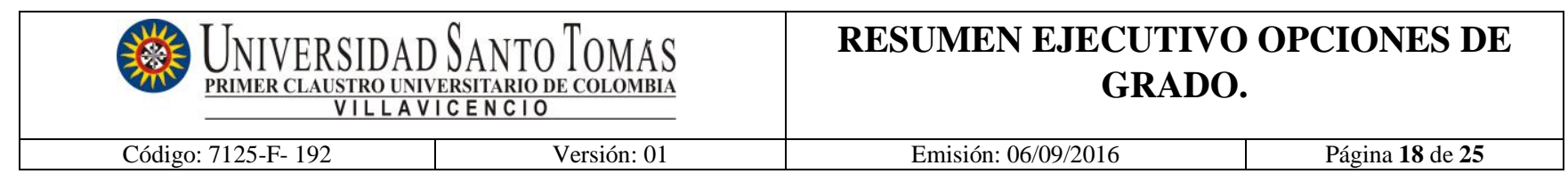

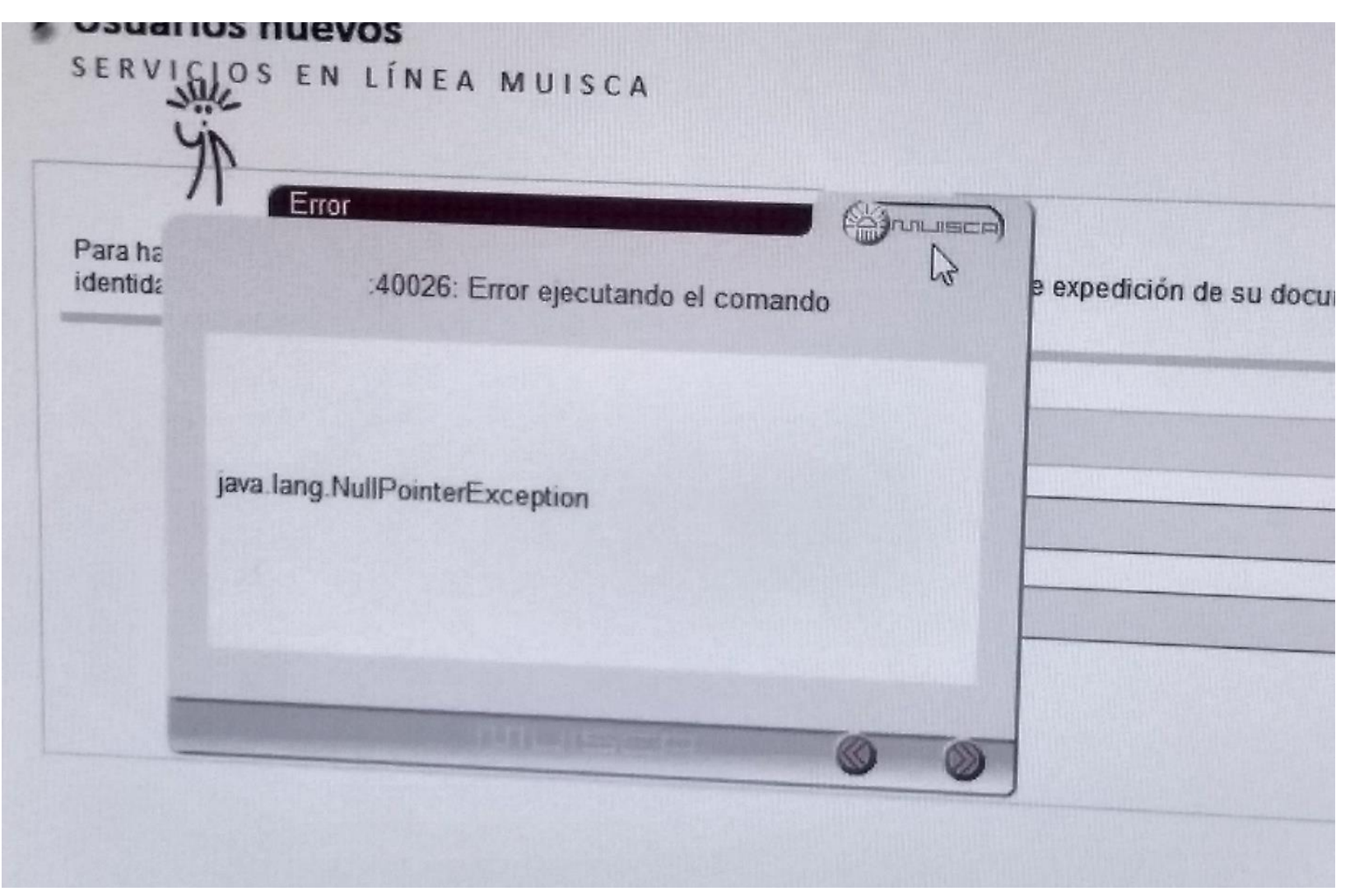

Ilustración 6. Error habilitando cuenta de usuario 


\begin{tabular}{|c|c|}
\hline 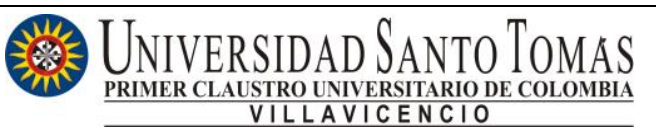 & $\begin{array}{l}\text { RESUMEN EJECUTIVO OPCIONES DE } \\
\text { GRADO. }\end{array}$ \\
\hline ligo: 7125-F- 192 & Emisión: 06/09/2016 \\
\hline
\end{tabular}

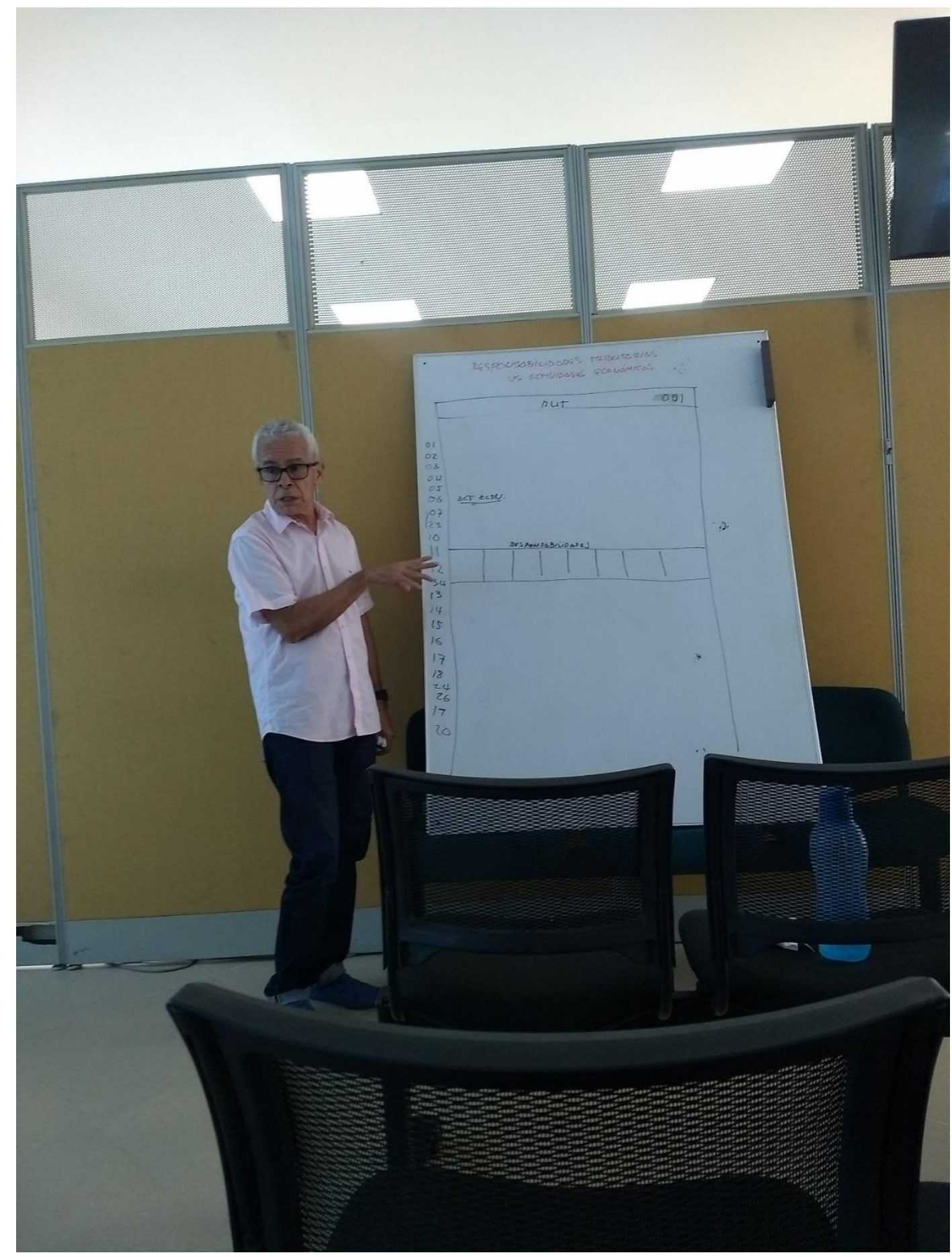

Ilustración 7. Capacitación Responsabilidades RUT. 


\begin{tabular}{|c|c|c|c|}
\hline 潘今 UNIVER & TOMAS & \multicolumn{2}{|c|}{$\begin{array}{l}\text { RESUMEN EJECUTIVO OPCIONES DE } \\
\text { GRADO. }\end{array}$} \\
\hline Código: 7125-F- 192 & Versión: 01 & Emisión: 06/09/2016 & Página 20 de 25 \\
\hline
\end{tabular}

\section{LOGROS ALCANZADOS Y RESULTADOS}

\section{Personales}

* Fortalecimiento de la confianza en mí mismo

* Aplicación en casos reales de los conocimientos tributarios adquiridos

* Mejora de la comunicación interpersonal

* Reforzar el trabajo en equipo

- Encontrar distintas soluciones a las dificultades

\section{Generales}

* Cumplir satisfactoriamente con el nivel exigido por el jefe de la división

* Aprender el funcionamiento de los SIE

* Colaborar a los funcionarios a cumplir satisfactoriamente con sus objetivos

* Ofrecer soluciones para evitar retrasos en atención

\section{CONCLUSIONES Y RECOMENDACIONES}

La experiencia en las empresas públicas o privadas ayudan a tener una visión de la realidad ya que algunos estudiantes no han tenido trabajo o no han trabajo en el área contable.

* Facilitar al practicante algún sustento para transporte y/o alimentación para poder cumplir con las exigencias de la entidad.

* Evitar enviar estudiantes a empresas donde no se apliquen conocimientos necesarios para la vida profesional para que el estudiante no solo cumpla un requisito de grado, sino que también se nutra en cuanto a conocimientos profesionales. 


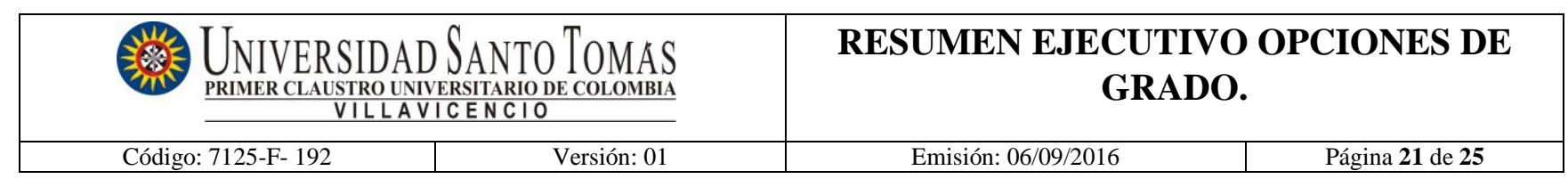

\section{REFERENTES BIBLIOGRAFICOS, VIDEOS, WEB Y CON EXPERTOS}

* Gildardo Vanegas, Profesional en Administración de empresas, Especialización en Administración, Funcionario de la UAE DIAN.

* Power Point - Entidades Sin Ánimo de Lucro (ESAL)

* Power Point - Nuevos obligados a presentar Declaración de Ingresos y Patrimonio

* Concepto Unificado 0481 de 2018.

https://www.dian.gov.co/normatividad/Documents/Concepto_unificado_ESAL_No_481_ 27042018.pdf

* Resolución Número 00001928 de marzo 2018 


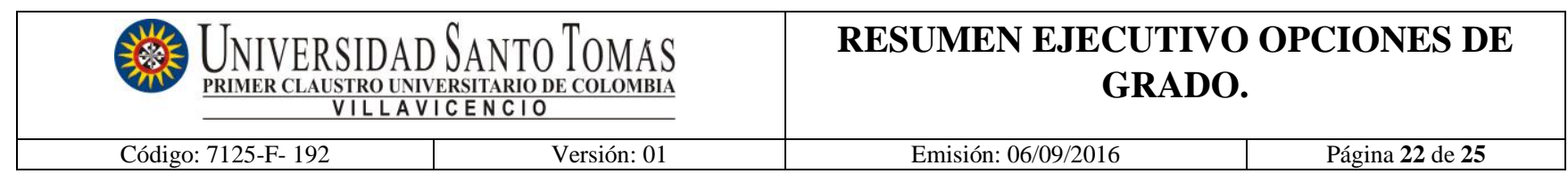

ANEXOS

En el CD se entregan los siguientes anexos

- Diapositivas de referencias

- Resolución. 


\begin{tabular}{|c|c|}
\hline 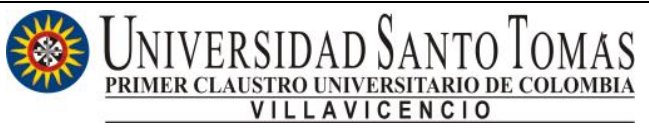 & $\begin{array}{l}\text { RESUMEN EJECUTIVO OPCIONES DE } \\
\text { GRADO. }\end{array}$ \\
\hline Código: 7125-F- 192 & Emisión: 06/09/2016 \\
\hline
\end{tabular}

Entregado en Villavicencio, Meta a los días del mes de del año 2018.

Quien entrega:

OSCAR ALIRIO BENITO MOLINA

CC. 1.121.938.233

Nombre y Documento

Calidad: Estudiante 


\begin{tabular}{|c|c|c|c|}
\hline 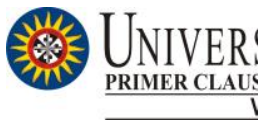 & $\begin{array}{l}0 \text { TOMAS } \\
0 \\
0\end{array}$ & \multicolumn{2}{|c|}{$\begin{array}{l}\text { RESUMEN EJECUTIVO OPCIONES DE } \\
\text { GRADO. }\end{array}$} \\
\hline Código: 7125-F- 192 & Versión: 01 & Emisión: 06/09/2016 & Página 24 de 25 \\
\hline
\end{tabular}

TABLA DE VALORACION

\begin{tabular}{|c|c|c|c|c|}
\hline \multirow[t]{2}{*}{ Aspecto } & \multicolumn{4}{|c|}{ Nivel de cumplimiento } \\
\hline & Muy Alto & Alto & Medio & Bajo \\
\hline $\begin{array}{l}\text { Contiene todos los elementos } \\
\text { establecidos en el documento } \\
\text { "Resumen Ejecutivo Académico". }\end{array}$ & & & & \\
\hline $\begin{array}{l}\text { Se hace explícito los resultados } \\
\text { de manera clara y concreta. }\end{array}$ & & & & \\
\hline $\begin{array}{l}\text { Hay coherencia en la } \\
\text { construcción del documento. }\end{array}$ & & & & \\
\hline $\begin{array}{l}\text { Evidencia revisión de fuentes de } \\
\text { información. }\end{array}$ & & & & \\
\hline $\begin{array}{l}\text { Cumplimiento de condiciones de } \\
\text { entrega. }\end{array}$ & & & & \\
\hline
\end{tabular}

Se considera aceptado el documento cuando cumpla con todos los aspectos en su totalidad. 


\begin{tabular}{|c|c|}
\hline 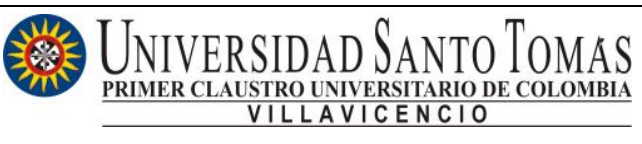 & $\begin{array}{l}\text { RESUMEN EJECUTIVO OPCIONES DE } \\
\text { GRADO. }\end{array}$ \\
\hline Versión: 01 & Emisión: 06/09/2016 \\
\hline
\end{tabular}

Validado en a los días del mes de del año

Quien Valora el documento:

Nombre:

Documento:

Calidad: Docente Coordinador del proceso.

Nombre:

Documento:

Calidad: Coordinador Opciones de Grado.

QUIEN RECIBE EN LA FACULTAD PARA ARCHIVO:

Nombre:

Documento:

Cargo:

Fecha: 